\title{
360 Degree Feedback as a Technique of Performance Appraisal: Does it Really Work?
}

\author{
Md. Sajjad Hosain \\ Senior Lecturer, Department of Business Administration, Uttara University, Dhaka, BANGLADESH \\ *E-mail for correspondence: sajjadhossain@uttarauniversity.edu.bd
}

\begin{abstract}
The overall performance of a firm is mostly dependent on the individual and group performance of the organization. Performance appraisal is a continuous process of monitoring, managing and correcting the individual performance of entire workforce. 360 degree feedback is considered as one of the yardsticks of performance appraisal process which reduces and changes the traditional supervisor based appraisal method. This qualitative study has been carried out to discover whether 360 degree feedback is effective as the part of overall performance appraisal process highlighting results from previously conducted researches and also, made an attempt to deliver some recommendations about how to use it more efficiently. Results from the previous studies revealed that 360 degree feedback is an effective method for appraising employee performance despite of having a few drawbacks and can be more useful if integrated with some traditional methods.
\end{abstract}

Keywords: 360 Degree Feedback, Performance, Performance Appraisal, Employee, Firm, Evaluation, Employee Development JEL Classification Code: L2

\section{INTRODUCTION}

The optimum effectiveness and efficiency of a firm neither depend on its financial resources, nor using the latest technology, even its not the best strategy, rather it is determined by the extent to how well it is using its dedicated, motivated and efficient employees (Hosain, 2015). To be competitive, an organizations need to help adapt and evolve its human resource to the highest possible extent. Because of this, first, a firm first should try to know how its people are currently performing and if there is any necessity to change. This is where 360 degree feedback is playing a vital role in its ability to provide structured, in-depth information about the current performance and the requirements of an employee in future to enable detailed and relevant development plans to be formulated (Baroda et al., 2012). Traditionally, the feedback used to be taken from only immediate supervisor. Later, due to the spread of flattened structures of firms and the increasing necessity to respond demand, 360 degree feedback appraisal method was introduced to help employees with the information needed to deal with rapid change and to combine individual talent with organizational objectives. At present, many reputed firms around the globe fully utilizing it as part of specific competencies and requirements to meet their objectives. Some of the firms have even gone further up and liked this process to performance appraisal and succession planning (Baroda et al., 2012).

\section{LITERATURE REVIEW}

Traditionally, performance analysis of an employee was mainly done form one feedback source-his/her immediate supervisor. 360 feedback is relatively a new concept that involves a variety of sources such as peers, clients, subordinates, supervisors and even from self ratings (self-evaluation). This information are used to identify the strengths and weaknesses of an employee to maximize productivity and also, to help him/her understand professional position regarding those competencies (Baroda et al., 2012). Mostly, in 360 feedback method include more than one party - peers, supervisors, subordinates and customers. The survey often includes computerized and web based responses that are summarized in to individual reports to be rated. When they meet the supervisors, they share the information that they feel pertinent for their own convenience (Baroda et al., 2012).

Therefore, the technique is the matter of debate to the practitioners and researchers. One study found significant correlations between 360 degree feedback and conventional performance ratings (Beehr et al., 2001) while another study concluded that multi-source feedback leads to "generally small" performance improvements on subsequent ratings (Smither et al., 2005). However, integrating 360 degree appraisals with behavioral competencies improves the rating reliability 
highlighted in one study, the competency based 360 degree feedback assessments were strongly predictive of how the managers performed in a subsequent assessment center (Hagan et al., 2006). However, Haworth (1998) and Pfau \& Kay (2002) found that companies using 360 degree feedback have comparatively lower market value, perhaps due to the methodological complications.

Most of the above literatures suggest that firms should carefully assess the tentative costs of the method, focus on the feedbacks very clearly on concrete goals, extensively train the personnel who are providing and receiving feedbacks and not rely exclusively on 360 feedbacks. The company using this technique should also ensure that it is productive, unbiased and goal oriented. Like a full circle, 360 feedback help managers to get a transparent view of impact they are having in their working space. While the technique has gained popularity over the past $10-15$ years among the managers for staff development, it can also serve as a communication device for them to provide information about how well they are responding. Moreover, when employees are allowed to respond about how their managers' style is being perceived empowering results that take place (Bailey, 2005 \& Fisher, 2004).

\section{Reasons for using 360 degree feedback method over single feedback}

360 degree feedback has obviously several significant reasons over single-rated feedback methods. Rather than relying on the perceptions or feedback of single individual, the multi-rated feedback derives multiple perceptions from different angles which bring a broader overview of an employee's performance. Those working with the employee, along with the supervisor are generally provide a more comprehensive picture of an employee's behavior or performance especially when the supervisor does not have the opportunity to oversee all areas of an employee's performance (Maylett, 2009). Proving information to managers about how they are viewed by direct subordinates, peers and clients can gain as much honest feedback as is necessary than an individual self-perception.

A second alternative cause for the adoption of 360 degree feedback is the desire to expand formal appraisal process by making such feedback more evaluative, linking it directly to the managers evaluation. One recent experience suggests that there are pressures to make 360 degree feedbacks evaluative as companies want to get more to their investments' worth (Baroda et al., 2012).

According to Kouzes and Pozner (1993), 360 degree feedback is one of the most powerful mechanisms in the field of performance appraisal process. It involves legitimacy, reliability and responsibility in overall process (Basu, 2015). London and Beatty (1993) suggest that 360 degree feedback can be a powerful organizational intervention to increase awareness of the importance of aligning behavior, work unit performance and customer expectations; as well as increasing participation in leadership development and work effectiveness. They also stated that it recognizes the complexity of management and the value of input from various sources.

\section{Are the techniques free from drawbacks?}

There are some significant disadvantages of using 360 degree feedback for appraisal purpose. As many organizations and managers operate in poor feedback environments, the first exposure to this method may be accompanied by some degree of angst on the part of both organization and employee (Maylett, 2009). When employees can anticipate that feedback they receive will be used purely for their own developmental benefit they tend to be more receptive to the feedback provided. Rather than receiving the feedback from a defense posture, they are more apt to accept the feedback as a "gift" from those the influence (Maylett, 2009). When 360 degree feedback has administrative consequences like pay rise, promotions, bonuses or possible layoffs, employee may be more aggressive to it rather than accepting it as it is. On the other hand, feedback providers tend to be less likely to give honest, impartial and fair feedback if they know that it might affect someone's pay or promotion they are close to.

Employees may resist and try to sabotage such program. For example, in case of upward feedback, implicit or even explicit deals may be struck with subordinates to give high ratings in exchange for high ratings and such manipulation is less likely when feedback is provided strictly for developmental purpose (Baroda et al., 2012). Research has demonstrated that when ratings become evaluative rather than purely developmental, some raters (up to 35\%) change their ratings (Pfau, Kay, Nowak \& Ghorpade, 1998).

The employee may be in a world of fear when the appraisal is negative for him or her. He/she might get a feeling that all the employees are ganging up against to frame him/her (Baroda et al., 2012).Lastly, anonymity and privacy breach may become a major issue since multiple parties are involved in 360 feedback process. It is likely that evaluators may discuss an employee's appraisals openly and violate his/her privacy (Baroda et al., 2012).

\section{Degree FeEdback: Some Recommendations TO MAXIMIZE EFFECTIVENESS}

A number of companies have used or are using 360 degree feedback for their performance appraisals and have derived a lot of benefits while others cannot. This is not only due to the weaknesses inherited on the technique but also on the way of using and who are using it. However, firms may adopt the following tips to maximize the benefits from this technique and minimize the risks involved. 
Identification of the differences in use and purpose: Understanding the feedback scores might differ depending on the purpose, the user should know how to best use and for what purpose; and also how to interpret the scores. Trainings might be provided to employees to enhance self-awareness to minimize fluctuations in ratings and also to get more accurate self-ratings (Baroda et al., 2012).

Communicating the purpose and process: Employees should know the intended purpose before administering the assessment and how the results will be interpreted (Maylett, 2009). Proper communication at the beginning will make the employee relaxed and pre-prepared. For this purpose, an orientation to all the employees can be given about the assessment and implementation of the 360 degree appraisal process.

Pilot study: Introducing a pilot study on a group of 30 to 50 employees prior to implementing company-wide appraisal may be done. It would help refining and identifying any hidden disadvantages of the technique and instruments. The pilot survey will also provide a message of what is expected and how it would be done to the overall organization.

Taking time before taking any action: Before taking any action, a company should wait a minimum period of time to allow people become familiar with the process and feeling more comfortable with the feedback (Maylett, 2009).

Selection of appropriate raters: It is often more realistic for employees to chose their own raters with development feedback rather than with appraisal (Maylett, 2009). Most of the weaknesses of 360 degree feedback technique can be solved by selecting raters on behalf of the employee rather than giving freedom to select their own. It is also important to ensure that the employees being rated are in regular interaction with the raters and provide accurate feedback in terms of performance.

Using small but relevant rater groups: The number of people involved in providing feedback should be considered. Each employee, including managers, might be required to complete multiple evaluations. Since time important, number of assessments and employees may be taken in to consideration (Maylett, 2009).

Consideration and communication of rating scale: In general, a 7 point Likert scale is more effective than a 5 point scale to allow greater differentiation in scores. The ratings should also be provided with proper directions and guidelines regarding what each scale means and how the levels of performance should be rated. The overall result will be a common understanding of related degree of various levels of performance.
Short survey: The survey questionnaire should be designed in such a way that it could be completed in 15 minutes or less. DecisionWise (2008), in one of its studies, found that there should be approximately 45 to 55 questions. If it is longer, the raters tent experience fatigue which results in more "good" or "average" range. The shorter the questionnaire, the more accurate feedback tend to be. The questions should also be concise.

Customized survey: Survey questions should be designed for specific purpose. Organizations should consider a customized, purpose-specific survey rather than a standard, general one.

Proving score per question: Many performance appraisals design a series of identical questions under one category or group. If "communication" is taken as an example that has many components like oral, written, verbal and non-verbal, scores should be provided on the basis of each component, not an overall score for only communication. This will be easier for an employee to understand which area of communication is being addressed by the surveyor. It will help to get more useful and reliable data thus make it easy for the rater to evaluate accurately and easily.

Integrating 360 degree feedback with training and development process: Once the 360 degree feedback method is considered, it should be integrated with other training and development initiatives. Proving feedback to suggest changes without training or assistance will result in lowering motivation and fear among the employee who are to be evaluated.

Integrating in to organizational culture: Last but not the least, it should be integrated in to the organizational culture as part of regular and systematic process. It will help the employees used to the technique and remove confusion, fear and resistance thus increasing confidence.

\section{ConCluding Remarks}

The 360 degree feedback has both advantages and disadvantages. It is important to note that 360 degree feedback for performance and 360 degree feedback for development are likely to produce completely different results (Maylett, 2009). They should not be used interchangeably. To get the optimum result, organizations should use both 360 degree feedback and traditional performance evaluation methods together. Using different feedback methods will provide a more complete and accurate picture of overall employee performance. 360 degree feedback technique has the ultimate potential to provide precious insight for both the firm and the employees if properly utilized. 


\section{REFERENCES}

Bailey, A. (2005). "How to square the circle on 360 degree feedback". Personnel Today, pp.17-21.

Baroda, S., Sharma, C. and Bhatt, J. K. (2012). “360 Degree Feedback Appraisals- An innovative approach of Performance Management System". International Journal of Management $\mathcal{E}$ Information Technology, Vol. 1, No. 2, pp. 53-66.

Basu, T. (2015). "Integrating 360 degree feedback in to performance appraisal tool and development process". IOSR Journal of Business and Management, Vol. 17, No.1, pp. 50-61. DOI: 10.9790/487X-17135061.

Beehr, T. A., Ivanitskaya, L., Hansen, C. P., Erofeev, D. \& Gudanowski, D. M. (2001). “Evaluation of 360 degree feedback ratings: relationships with each other and with performance and selection predictors". Journal of Organizational Behavior. Vol. 22, No.1, pp. 775-788.

DecisionWise Leadership Intelligence Inc. (2008). Evaluating Employee Performance: Multisource feedback and traditional performance appraisal provide a bigger picture. Available from DecisionWise Website: http:/ / www.decwise.com

Fisher, B. A. (2004). "Listen to what's really going on". Supervision, pp. 9-11.

Hagan, C. M., Konopaske, R., Bernardin, H. J. \& Tyler, C. L. (2006). "Predicting assessment center performance with 360-degree, top-down, and customer-based competency assessments". Human Resource Management, Vol. 45, No.3, pp. 357 - 390. DOI: 10.1002/hrm. 20117

Haworth, S. (1998). "The dark side of multi-rater assessments". HR Magazine, Vol. 43, pp. 106-111.
Hosain, M. S. (2015). "Adoption of Proper HRM Practices: A Technique for Retaining Employees and Increasing Firm Performance?" Scholar Journal of Business and Social Science, Vol. 1, No. 1, pp. 1-14.

Kouzes, J. H. \& Pozner, B. Z. (1993). “Credibility - How leaders Gain and Lose it, Why people Demand It". San Francisco: Jossey - Bass publishers, pp. 97-98.

London, M. \& Beatty, R. W. (1993). “360 degree feedback as competitive advantage". Human Resource Management, Vol. 23, No. 4, pp. 77-82.

Maylett, T. (2009). “360 degree feedback revisited: The Transition from development to appraisal". Decision-wise Leadership Intelligence, pp. 1-8. Retrieved from www.decwise.com.

Pfau, B. and Kay, I. (2002). “Does 360-degree feedback negatively affect employee performance?" HR Magazine, Vol. 47, pp. 54-59.

Pfau,B., Kay,I., Nowak, K. \& Ghorpade,J. (1998). “Does 360-degree feedback negatively affect company performance?" Managing Human Resources, Vol. 32, No. 3, pp. 86-94.

Smither, J. W., London, M., \& Reilly, R. R. (2005). “Does performance improve following multi-score feedback? A theoretical model meta analysis and review of empirical findings", Personnel Psychology, Vol. 58, pp. 33-66.

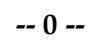

